Mit Ecodesign zu ökointelligenten Produkten

\title{
Wettbewerbsvorteile durch ökologische Produkte
}

\author{
Durch das Auffinden von Umweltschwachstel- \\ len und das konkrete Gestalten von Produkten \\ mit Ecodesign können erfolgreich ökointelli- \\ gente Produkte entwickelt werden. Die Erfah- \\ rungen aus verschiedenen Fallbeispielen liefern \\ konkrete Hinweise für die Umsetzung in \\ Produktion und Vermarktung. \\ Von Wolfgang Wimmer
}

Ö kointelligente Produkte sind kein Luxus, sondern Notwendigkeit. Kunden erwarten zunehmend von Produkten, dass diese die Umwelt weniger belasten und keine kritischen Stoffe enthalten. Zudem verlangen aktuelle Gesetze und Verordnungen von Unternehmen, dass die Umweltbelastungen der Produkte insgesamt reduziert werden. Produkthersteller sind deshalb aufgefordert, neue und innovative Konzepte im Sinne von Ecodesign zu realisieren.

Wie aber soll ein Unternehmer dabei vorgehen? Wo soll man beginnen und wie kann man Ecodesign konkret auf Produkte anwenden? Und zuletzt: Was hat ein Unternehmen davon?

Eine einfache Überlegung zu Beginn: Betrachten wir einen konventionellen Fernseher und messen wir den Energieverbrauch. Das Ergebnis zeigt einen Energieverbrauch von etwa 65 Watt im Betriebs- und sechs Watt im Stand-by Modus. Als nächstes bestimmen wir das Nutzungsszenario - im Durchschnitt könnten das etwa vier Stunden fernsehen und 20 Stunden Stand-by pro Tag sein. Eine marktübliche Nutzungsdauer beträgt sieben Jahre für einen Fernseher. Damit ergibt sich ein Gesamtenergieverbrauch von 970,9 Kilowattstunden, etwa ein Drittel davon wird durch den Stand-by Modus verursacht. Nehmen wir nun weiter an, dass der Hersteller 300.000 Geräte dieses Typs auf den Markt bringt. Dann beträgt der Flottenverbrauch dieses einen Fernsehers 292 Gigawattstunden. Immerhin 92 Gigawattstunden davon macht der Verbrauch im Stand-by aus, also Energieverbrauch, der anfällt, ohne einer einzigen Stunde Fernsehens. 92 Gigawattstunden, das ist die Dimension eines kleinen Kraftwerks.

\section{Nur wenige Produktverbesserungen}

Übrigens verbraucht auch eine elektrische Zahnbürste, ein Ladegerät für das Mobiltelefon, ein DVD-Player, ein Radiowecker zwischen 1,5 und 2,5 Watt pro Stunde im Stand-by, ein Tin- tenstrahldrucker oder eine Waschmaschine können mit bis zu 20 Watt pro Stunde zu Buche schlagen.

Zurück zum Fernseher. Dieser hat vielleicht 200 Euro in der Anschaffung gekostet. Bei einem angenommenen Stromtarif von 0,2 Euro pro Kilowattstunde kostet der Betrieb während der gesamten Lebensdauer 194 Euro, also noch einmal so viel wie die Anschaffung - 61 Euro sind für den Stand-by erforderlich.

Geht das auch anders? Sicher sogar. Mit weniger Kosten und weniger Umweltverbrauch und damit mit weniger Umweltbelastung. Ökointelligente Produkte durch Ecodesign sind gefragt (Wimmer 2004).

Wenn Sie sich denken, dass das nicht neu ist und vor 20 Jahren schon diskutiert wurde, so ist das richtig. Genauso richtig ist aber auch, dass bisher keine Lösungen angeboten werden. Die oben beschriebenen Messungen wurden erst vor kurzem an einem modernen TV-Modell durchgeführt und keineswegs an einem TV-Fossil. Neu ist, dass es so nicht mehr weitergehen können wird. Die EU-Kommission hat die sogenannte Energyusing-Product-Directive, kurz EuP-Richtlinie, herausgegeben, die für 14 Produktkategorien Beschränkungen des Energieverbrauchs verlangt (Europäische Kommission 2005). Stand-by Verbrauch ist eine dieser Kategorien. Im März 2007 werden dafür Maßnahmen zur Umsetzung veröffentlicht werden. Erfüllt ein Hersteller die Vorgaben nicht, bekommt er kein CE-Kennzeichen und kann seine Produkte schließlich nicht mehr in der EU verkaufen. Es wird also endlich ernst.

\section{Die Umweltschwachstelle des Produktes finden}

Auf der anderen Seite haben innovative Firmen längst erkannt, dass Ecodesign Wettbewerbsvorteile bringt und setzen schon längst auf die Integration von Umweltüberlegungen in neuen, besseren Produkten. Dabei ist allerdings nicht immer im Vorhinein klar, dass der Energieverbrauch in der Nutzung die Schwachstelle ist.

Um Ecodesign erfolgreich umzusetzen, gilt es also zunächst die Umweltschwachstelle des Produkts zu finden, oder, wie die EuP-Richtlinie es formuliert, ein Umweltprofil des Produktes zu erstellen. Dabei müssen alle Lebensphasen des Produktes analysiert werden und die Umweltbelastungen in den einzelnen Lebensphasen berechnet werden. Üblicherweise unterscheidet man die Lebensphasen Rohstoffgewinnung, Herstellung, Distribution, Nutzung und Nach-Gebrauch.

Die Umweltbelastungen zu ermitteln ist nicht so einfach. Das Gewicht kann man mit einer Waage messen, die Länge $\rightarrow$ 


\section{„Um Ecodesign erfolgreich umzusetzen, müssen alle Lebensphasen des Produktes analysiert und so die Umweltschwachstelle des Produktes gefunden werden. "}

mit einem Maßband. Wie aber kann man Umweltverbrauch messen? Die internationale Konvention dazu ist in der Norm ISO 14040 festgelegt (Internationale Organisation für Normung 2006). Dort wird geregelt, in welchen Wirkungskategorien Umweltverbrauch im Rahmen einer Ökobilanz gemessen werden soll. Wirkungskategorien sind unter anderem das Treibhauspotenzial gemessen in Kohlendioxid-Äquivalenten oder die Versauerung gemessen in Schwefeldioxid-Äquivalenten.

Eine andere Vorgangsweise wäre, das Umweltprofil eines Produktes zu erarbeiten, indem man die Energieverbräuche entlang der fünf Lebenszyklusphasen ermittelt. Also die Energie zur Gewinnung der Rohstoffe, die Energie zur Verarbeitung derselben zum fertigen Produkt, die Energien für den Transport und die Herstellung der Verpackung, die Energie für den Betrieb des Produkts in der Nutzung und die Energie für die Sammlung, Materialtrennung und Wiederverwertung im Fall des Recyclings.

\section{Produktanalyse mit dem Fokus Energie}

Wem ist bewusst, dass der Energieverbrauch zur Gewinnung von Aluminium aus Erz bei etwa 55 Kilowatt pro Kilogramm liegt? Hingegen benötigt die Gewinnung von Aluminium aus Altmetallen, also eingeschmolzenem Schrott, um den Faktor 20 weniger Energie.

Die Herstellung von einem Kilogramm bestückter Leiterplatte inklusive aller Bauteile benötigt circa 1000 Kilowattstunden pro Kilo. Der Grund dafür sind vor allem die in den Bauteilen verwendeten Edelmetalle wie Palladium, Gold oder Silber. Auch dazu eine Größenordnung: Aus 20 Kubikmeter bestückten Leiterplatten lässt sich im Recycling etwa ein Kilogramm Gold gewinnen. Vorausgesetzt, man verfügt über die richtige Technologie und die entsprechenden Verfahren. Aus diesen Zahlen kann man erkennen, wie richtig die Entscheidung der EU war, die WEEE-Richtlinie einzuführen, die die Kunden seit dem 15. August 2006 verpflichtet, ihre alten Elektrogeräte nicht mehr im Restmüll zu entsorgen sondern diese an den Sammelstellen zurückzugeben. Gleichzeitig sind die Hersteller verpflichtet, eine dem Stand der Technik entsprechende stoffliche Verwertung zu finanzieren (Europäische Kommission 2003b).

Soweit so gut, aber was kann nun bei vorliegendem Umweltprofil und den erkannten Umweltschwachstellen getan werden, um Produkte mit Ecodesign zu verbessern? Dazu sollen zwei
Fallbeispiele, eine Vorgangsweise in sechs Schritten zur Integration von Ecodesign in einem Unternehmen sowie weitere hilfreiche Tools vorgestellt werden.

\section{Softwaretools für nachhaltiges Produktdesign}

Unsere Arbeiten mit dem Büromöbelhersteller Steelcase Inc. begannen vor nunmehr drei Jahren, als sich Andre Malsch aus der Entwicklungsabteilung in Straßburg bei uns meldete und sich für unser Softwaretool, den Ecodesign-PILOT, interessierte. Er wolle Sustainability in der Firma umsetzen - so sein Wunsch damals. Mittlerweile hat sich viel getan.

In Stichworten lässt sich der Weg von Steelcase Inc. mit Training, Produktanalyse, Produktverbesserung und gezielter Umweltkommunikation beschreiben.

Wir haben mit Workshops begonnen, die Entwicklungs- und Konstruktionsabteilung über Ecodesign zu informieren. Von der damaligen Haltung „Nein, nicht auch das noch“ zu der heute fix im Produktentwicklungsprozess verankerten Berücksichtigung von Umweltüberlegungen haben drei Dinge wesentlich beigetragen. Erstens die CEO-Entscheidung, voll auf Sustainability und Ecodesign zu setzen. Zweitens die konsequente Ökobilanzierung aller neu bei Steelcase hergestellten Produkte und drittens die Bereitstellung von Umweltwissen für die Produktentwickler durch die eigens entwickelten Softwaretools Furniture-PILOT und Material-Selector und durch die Entwicklung von Golden-Design-Rules. Dabei wurde Steelcase von uns in allen drei Elementen begleitet. Am Ende dieses Prozesses stand die Neuentwicklung des Bürodrehstuhls THINK. Das Ergebnis kann sich sehen lassen. THINK hat gemessen am Vorgängermodell nicht nur ein deutlich geringeres Gewicht, sondern auch 30 Prozent weniger Teile bei gleicher Funktionalität und einen Anteil an Recyclingstoffen von über 40 Prozent. Zudem wurden bewusst problematische Substanzen weggelassen, die Produktion vereinfacht und in der Summe die Umweltbelastungen des Produktes stark reduziert. Für den Bürostuhl wurde eine Ökobilanz erstellt und die erzielten Umwelteigenschaften in Form einer Produktumwelterklärung dokumentiert. Zu guter Letzt ist THINK am Ende seines Produktlebens in fünf Minuten mit Standardwerkzeugen zerlegbar. Dies war als wesentliche Voraussetzung für den nächsten Schritt der Produktrücknahme gefordert.

Steelcase hat Ecodesign voll in die Produktentwicklung integriert, erstellt für jedes Produkt eine Ökobilanz nach ISO 14040 und dokumentiert die erzielten Umwelteigenschaften für jedes neue Produkt mit einer Produktumwelterklärung.

\section{Umweltanalyse als Ausgangspunkt}

Siemens Transportation mit Sitz in Wien, Graz und Erlangen wird seit zwei Jahren von uns betreut. Ziel der Kooperation ist die Entwicklung von Greenline, eines neuen Fahrzeugkonzepts für eine Metro der Zukunft unter spezieller Berücksichtigung von Umweltüberlegungen. Auch bei Siemens Transpor- 
tation stand zunächst die Umweltanalyse im Vordergrund. Es wurde eine Ökobilanz für das Referenzprodukt, die neue Metro von Oslo/Norwegen durchgeführt. Ein durchaus umfangreiches Projekt, bei dem mehr als 60.000 verschiedene Komponenten analysiert werden mussten, die im Metrozug, bestehend aus drei Wagen bei einem Gesamtgewicht von 100 Tonnen, verbaut sind.

Die Ergebnisse der Ökobilanz zeigen das klassische Umweltprofil eines nutzungsintensiven Produkts mit einer Lebensdauer von 35 Jahren und einer Jahreskilometerleistung von 120.000 $\mathrm{km}$. Als Ansatzpunkte für Umweltverbesserungen ergeben sich daraus eine effizientere Heizung- beziehungsweise Klimatisierung etwa durch Abwärmenutzung, eine Wirkungsgradverbesserung im Antriebssystem oder aber auch konsequenter Leichtbau im Wagenkasten.

Im nun folgenden Schritt des laufenden Projekts geht es darum, die effizientesten Verbesserungsoptionen auszuwählen und in einem neuen Konzept umzusetzen. Als ein Zwischenergebnis wurde unter anderem für die Metro Oslo eine Produktumwelterklärung erstellt, die die aktuelle Umweltsituation beschreibt. Das Projekt hat Anfang Dezember einen der weltweit ausgeschriebenen Siemens-Umweltpreise gewonnen.

\section{In sechs Schritten zum Ecodesign}

- Umweltindikatoren entwickeln. Als erster Schritt im Ecodesign Prozess steht die Umweltbewertung und die nachfolgende Festlegung von Umweltindikatoren (EPIs - Environmental Performance Indicators). Dabei handelt es sich um Messgrößen, die einen unmittelbaren Umweltbezug haben, beziehungsweise am direktesten die Umweltbelastungen des Produktes entlang seines Lebensweges steuern. Das könnte beim Bürostuhl beispielsweise das Gewicht, der Energieverbrauch in der Produktion oder die erzielbare Recyclingrate sein.

I Tools und Datenbank erstellen. Als Ergebnis der Umweltbewertung ist im zweiten Schritt firmeneigenes Umweltwissen etwa in Form der Entwicklung von maßgeschneiderten Tools und Datenbanken aufzubauen. Diese dürfen keineswegs kompliziert sein, sondern sollten unmittelbar in den Entscheidungen der Produktentwicklung die erforderliche Unterstützung bringen.

- Ecodesign Training durchführen. In Workshops und Seminaren sollten das Verständnis der Zusammenhänge und der Bezug zur vielfach neuen Thematik Umweltauswirkungen und ökologische Gestaltungsrichtlinien thematisiert werden.

I Green Flagship Produkte entwicklen. Den definierten Umweltindikatoren, dem erforderlichen Umweltwissen und der notwendigen Ausbildung sollte als nächster Schritt die Entwicklung eines Produktes folgen, bei dem ganz bewusst die Umwelteigenschaften im Vordergrund stehen, um ein Green Flagship Produkt zu entwickeln. Die Erfahrungen daraus sollen für die Entwicklung aller anderen Produkte genutzt werden.
I Umwelteigenschaften kommunizieren. Nachdem umweltoptimierte Produkte vorliegen, sollte eine entsprechende Umweltkommunikation nach außen vorbereitet werden, in der die erzielten Umweltverbesserungen dem Kunden dargestellt werden. Dafür wird idealerweise eine Produktumwelterklärung erstellt.

I Ecodesign im Produktentwicklungs- und Innovationsprozess implementieren. Im letzten Schritt sollten die gemachten Erfahrungen gesammelt und fix im Produktentwicklungs- beziehungsweise Innovationsprozess des Unternehmens verankert werden. Welche Checklisten sollten zu welchen Milestones oder Design Reviews bearbeitet werden? Wie fließen die Ergebnisse der Umweltbewertung in die Designentscheidungen ein? Wer ist für die Einhaltung und Messung der Umweltindikatoren verantwortlich? Wie wird der Einkauf für die Zulieferteile mit eingebunden? Diese und andere Fragen müssen gelöst und in die firmeninternen Prozesse integriert werden.

\section{Hilfe durch den Ecodesign-Pilot und -Assistent}

In Planungs- und Entscheidungsprozessen von Unternehmen wird zunächst die Situation analysiert und dann entsprechende Ziele für künftige Lösungen aufgestellt. Aufbauend auf einem solchen Zielsystem werden dann meist geeignete Maßnahmen entwickelt. Der Ecodesign-Pilot und -Assistent wurde vom Ecodesign Forschungsteam der Technischen Universität Wien entwickelt und hilft bei der Erstellung von Umweltzielen und Umweltmaßnahmen zur Produktoptimierung.

Der Ecodesign-Pilot bietet dazu eigene Lernseiten und praxiserprobte Checklisten, mit denen Produktentwickler einfach und rasch einen Zugang zu Ecodesign finden und Produkte zielgerichtet verbessern können (Wimmer 2001).

Zusätzlich wurde ein interaktiver Assistent zum EcodesignPilot erstellt, welcher aufgrund der Eingabe von wenigen wichtigen Lebenszyklusdaten die für eine Produktverbesserung infrage kommenden Ecodesign Verbesserungsstrategien ermittelt. $\mathrm{Zu}$ diesen wichtigen Daten gehört beispielsweise die Art und Menge der eingesetzten Materialien oder die Art und Weise der Nutzung.

Die Berechnungsmethode basiert auf mehreren einschlägigen Umweltbewertungsmethoden, weist diese allerdings nicht explizit aus, sondern schlägt direkt Produktverbesserungsstrategien vor.

Damit können die bedeutenden Umweltaspekte entlang des Produktlebenszyklus rasch erkannt, der Grundtyp des Produktes bestimmt, die Strategien ausgewählt und mögliche Maßnahmen zur Produktverbesserung evaluiert werden.

\section{Maßnahmen zur Produktverbesserung}

Für jede der nachfolgend angeführten Strategien gibt es im Ecodesign-PILOT eine eigene Checkliste, welche die für die $\rightarrow$ 
Strategien relevanten Ecodesign-Maßnahmen enthält. Für eine Verbesserung eines zu untersuchenden Produktes müssen nun aus den ausgewählten oder vom Assistenten vorgeschlagenen Strategien jene Maßnahmen gefunden werden, die optimalerweise zur Verbesserung des Produktes herangezogen werden sollen (1). Das sind:

- Zielgerichtete Materialwahl

- Materialeinsparung

- Verringerung des Energieverbrauchs in der Produktion

- Optimierung der Art und Menge an erforderlichen Hilfsund Betriebsstoffen in der Produktion

- Vermeiden von Abfällen in der Produktion

- Umweltfreundliche Beschaffung von Zukaufteilen

- Reduktion des Verpackungsaufwandes

- Reduktion des Transportaufkommens

- Vereinfachung der Bedienbarkeit durch neue Nutzungsweisen

- Optimierung der Funktionsweise des Produktes

- Steigerung der Produktlebensdauer

- Gewährleistung von hoher Umweltsicherheit

- Verbrauchsreduktion in der Nutzung

- Abfallvermeidung in der Nutzung

- Verbesserung der Wartbarkeit

- Verbesserung der Reparierbarkeit

- Erhöhung der Demontagefreundlichkeit

- Wiederverwendung von Produktteilen

- Wiederverwertung von Materialien

Für die Auswahl dieser Ecodesign Maßnahmen wird eine Konstruktionsbewertung des Produktes durchgeführt, um zu bestimmen, inwieweit das Produkt die in den Checklisten formulierten Ecodesign Handlungsanweisungen erfüllt.

Jene Maßnahmen, die für das Produkt noch nicht erfüllt wurden, sollen identifiziert und anhand der mitgelieferten Beispiele Ideen für das eigene Produkt gefunden werden.

Die Checklisten dienen in erster Linie dazu, im Team neue Ideen und mögliche Entwicklungen zu generieren. Wichtig dabei ist, dass bisherige Lösungen vorurteilsfrei hinterfragt werden, um so neue Wege zu finden. Als Mittel dazu haben sich die Checklisten sehr bewährt.

\section{Unternehmensspezifische Anpassung der Tools}

Nachdem die Anwendung des Ecodesign-Pilot sehr gute Ergebnisse brachte, bestand der Wunsch, die gesamte Methodik auf die firmenspezifischen Anforderungen zuzuschneiden.

Die Schritte einer solchen firmenspezifischen Anpassung des Ecodesign-Pilot lassen sich wie folgt angeben:

- Auswahl und Einschränkung auf die für das Produkt beziehungsweise die Produktpalette der Firma relevanten Ecodesign Maßnahmen

- Produktbezogene Formulierung der Ecodesign Maßnahmen samt spezifischer Darstellung und Erklärung

- Abbildung der verschiedenen Phasen des firmeneigenen
Produktentwicklungsprozesses samt Zuordnung der Ecodesign Maßnahmen zu diesen Phasen

- Zusammenstellung von maßgeschneiderten produktspezifischen Verbesserungsstrategien und den dazugehörigen Checklisten

- Generieren eines firmeneigenen Ecodesign-Pilot als Intranet-Lösung und Einsatz in der Produktentwicklung mit nachfolgender Evaluierung

- Einschulung der potenziellen Anwender in Form eines Workshops

Als erste Branchenlösung wurde der Elektro- und Elektronik-Pilot entwickelt. Damit wird eine Unterstützung bei der Umsetzung der WEEE und RoHS-Richtlinie geboten (2).

Ökointelligente Produkte zu entwickeln ist ein deutliches Zeichen für die Innovationskultur eines Unternehmens. Innovation und Weitblick für künftige Herausforderungen bestimmen die Wettbewerbsfähigkeit und damit den wirtschaftlichen Erfolg - gerade in einer sich stark ändernden Zeit.

\section{Literatur}

Europäische Kommission: Energy using Product directive. Establishing a framework for the setting of ecodesign requirements for energy-using products. EC/32/2005. Brüssel 2005.

Europäische Kommission: RoHS: Directive on the restriction of the use of certain hazardous substances in electrical and electronic equipment. EC/95/2003. Brüssel 2003.

Europäische Kommission: WEEE: Directive on waste electrical and electronic equipment, EC/96/2003. Brüssel 2003b.

Internationale Organisation für Normung: ISO 14040 - Environmental management. Life cycle assessment. Principles and framework, International Standard. Genf 2006.

Wimmer, W. / Züst, R. / Lee, K.-M.: Ecodesign Implementation - A Systematic Guidance on Integrating Environmental Considerations into Product Development. New York 2004

Wimmer, W. / Züst, R.: Ecodesign Pilot, Produkt-Innovations-, Lern- und Optimierungs-Tool für umweltgerechte Produktgestaltung mit deutsch/englischer CD-ROM. Zürich 2001.

\section{Anmerkungen}

(1) PILOT-online: www.ecodesign.at/pilot

(2) EEG-PILOT-online: www.ecodesign.at/pilot/eeg

\section{IAUTOR + KONTAKT}

Dr. Wolfgang Wimmer ist Professor an der Technischen Universität Wien, betreibt den Österreichischen Ecodesign Informationsknoten und ist seit 2005 Geschäftsführer der Ecodesign company GmbH mit Sitz in Wien, Seoul und Ottawa, mit der er Beratung zur Integration von Ecodesign im Unternehmen anbietet.

Dr. Wolfgang Wimmer, Technischen Universität Wien, Getreidemarkt 9, 1060 Wien, Österreich. Tel.: +43158801 307-44, E-Mail:wimmer@ecodesign.at, Website: www.ecodesign.at; www.ecodesign-company.com 
(c) 20I0 Authors; licensee IÖW and oekom verlag. This is an article distributed under the terms of the Creative Commons Attribution Non-Commercial No Derivates License (http://creativecommons.org/licenses/by-nc-nd/3.o/), which permits unrestricted use, distribution, and reproduction in any medium, provided the original work is properly cited. 